\title{
Caracterización clínica y tomográfica de pacientes hospitalizados con COVID-19
}

\author{
CRISTIAN DEZA E.*, MARÍA TERESA PARADA C.*, PATRICIA BITAR H.*, \\ JORGE DREYSE D.*, MATÍAS FLORENZANO V.*, CRISTIÁN IBARRA D.*, \\ JOEL MELO T.*, RODRIGO GIL D.* y CONSTANZA SALAS C.*
}

\section{Clinical and tomographic characterization of patients hospitalized with COVID-19}

Objective: To perform a clinical and imaging characterization in patients hospitalized for CO$V I D-19$ and to analyze whether there are risk predictors associated with greater severity of the condition. Method: Observational, retrospective study. Patients hospitalized with COVID-19 were included between April and July 2020. Demographic data, comorbidities, laboratory tests, tomographic pattern in thorax tomography (TC), therapies received, and type of respiratory support were recorded. In the statistical analysis to identify risk factors, we used Pearson's $\chi^{2}$ test or Fisher's test to compare categorical variables and Mann-Whitney test to compare continuous variables. Results: 164 patients were analyzed. Median age was 57 years (21 to 89). 111 patients (68\%) were male and a median of 7 days of symptoms prior to admission (1 to 23). 68 patients (41\%) have obesity (significantly higher in patients $<60$ years, $p=0.026)$, $56(34 \%)$ arterial hypertension (HT) and $43(26 \%)$ with diabetes mellitus. The predominant pattern in the admission CT scan was ground glass opacity (GGO) with "crazy paving" $(35 \%)$ and then pure GGO (28\%). Type of ventilatory support required was considered as an indicator of severity. 51 patients (31\%) require non-invasive ventilatory support (high-flow nasal cannula or NIMV) and 19 (11\%) invasive ventilation (IMV). The statistically significant predictor variables of severity were HT $(p=0.001)$, Diabetes Mellitus $(p=0.001)$ and Obesity. $(p=0.002)$. Conclusions: Patients hospitalized for COVID 19 with the highest risk of respiratory torpid evolution were obese, hypertensive and diabetic patients

Key words: SARS-CoV-2; COVID-19; Thorax Tomography Computed; Laboratories; Retrospective Studies; Comorbidities; Risk factors.

\section{Resumen}

Objetivo: Realizar una caracterización clínica y por imágenes a pacientes hospitalizados por COVID-19 y analizar si existen predictores de riesgo asociados con una mayor gravedad. Método: Estudio observacional, retrospectivo. Se incluyeron pacientes hospitalizados con COVID-19, entre abril y julio de 2020. Se registraron datos demográficos, comorbilidades, exámenes de laboratorio, tipo de compromiso en tomografía computada (TC) de tórax, terapias recibidas y tipo de soporte respiratorio. En el análisis estadístico para identificar factores de riesgo se utilizó test $\chi^{2}$ de Pearson o test de Fisher para comparar variables categóricas y test de Mann-Whitney para comparar variables continuas. Resultados: Se analizaron 164 pacientes. La mediana de edad fue de 57 años (rango 21 a 89). 111 pacientes (68\%) de género masculino y mediana de 7 días de síntomas previo al ingreso (rango 1 a 23). 68 pacientes (41\%) tienen obesidad (significativamente mayor en pacientes $<60$ años, $p=0,026$ ), 56 (34\%) hipertensión arterial (HTA) y 43 (26\%) diabetes. El patrón predominante en la TC de ingreso fue de vidrio esmerilado (VE) con "crazy paving” (35\%) y luego VE puro (28\%). Como indicador de gravedad se tomó en cuenta el tipo de soporte ventilatorio requerido: 51 pacientes (31\%) requieren soporte ventilatorio no invasivo (cánula nasal de alto flujo o VMNI) y 19 (11\%) ventilación invasiva (VMI). Las variables predictoras de gravedad, estadísticamente significativas, fueron: HTA ( $p=0,001)$,

* Clínica Las Condes. Santiago, Chile. 
Diabetes Mellitus ( $p=0,001)$ y Obesidad. $(p=0,002)$. Conclusiones: Los pacientes hospitalizados por COVID 19 con mayor riesgo de evolución tórpida, del punto de vista respiratorio, fueron los pacientes obesos, hipertensos y diabéticos.

Palabras clave: SARS-CoV-2; COVID-19; Tomografía axial computarizada de tórax; Exámenes de laboratorio; Estudios Retrospectivos; Comorbilidades; Factores de riesgo.

\section{Introducción}

La enfermedad producida por el nuevo coronavirus SARS-CoV-2, COVID-19, fue declarada pandemia por la Organización Mundial de la Salud (OMS) el 12 de marzo del año 2020. Al 30 de octubre de ese año, se han confirmado más de 45 millones de casos en el mundo con una tasa de letalidad del 2,6\% ${ }^{1}$. Según los datos actualizados del Ministerio de Salud de Chile (MINSAL) a la misma fecha, en nuestro país los casos confirmados superan los 500.000, con una tasa de letalidad del $2,8 \%$.

Esta patología produce una amplia variedad de compromiso respiratorio. Aproximadamente un $20 \%$ de los pacientes que la presentan requieren hospitalización y de estos un 17 a $35 \%$ requieren ser manejados en Unidades de Cuidados inten$\operatorname{sivos}^{2,3}$, principalmente debido a insuficiencia respiratoria hipoxémica.

Los pacientes portadores de patologías crónicas (especialmente Hipertensión arterial, Diabetes Mellitus y Obesidad), los de género masculino y los mayores de 65 años tienen una mayor probabilidad de cursar con la forma severa de la enfermedad ${ }^{4-6}$.

El objetivo de nuestro estudio fue realizar una caracterización clínica y tomográfica de pacientes hospitalizados con COVID-19 confirmado, ingresados en Clínica Las Condes. Se analizaron características demográficas, comorbilidades, exámenes de laboratorio, patrón en la tomografía computada (TC) de tórax, terapias recibidas y tipo de soporte respiratorio. Se analizó la existencia de variables predictoras de riesgo para mayor gravedad.

\section{Pacientes y Métodos}

Este estudio observacional y retrospectivo fue realizado en Clínica Las Condes e incluyó a pacientes hospitalizados con COVID-19 confirmado, entre los meses de abril y julio del año 2020 . Se incluyó a pacientes hospitalizados en servicio médico- quirúrgico, unidad de cuidados intermedios y unidad de cuidados intensivos; todos ellos fueron ingresados y luego seguidos en su evolu- ción por médicos especialistas en enfermedades respiratorias. El estudio cuenta con la aprobación del comité de ética de nuestra institución.

De acuerdo con las guías de la $\mathrm{OMS}^{7}$, la confirmación de infección por SARS-CoV-2 fue definida como un resultado positivo del examen de reacción de polimerasa en cadena (PCR en tiempo real) en muestras nasofaríngeas y orofaríngeas.

\section{Recolección de datos y variables analizadas}

Los datos fueron registrados por cada uno de los médicos tratantes en una planilla común, diseñada para tal efecto y luego fueron enviados al coordinador del estudio. Todos los médicos participantes se desempeñan como especialistas en enfermedades respiratorias en Clínica Las Condes.

Se registraron datos demográficos (edad y género), comorbilidades (obesidad, hipertensión arterial (HTA), cardiopatías, diabetes mellitus, enfermedades pulmonares crónicas, tabaquismo y cáncer activo (de menos de 1 año de diagnóstico), días de evolución (desde el inicio de los síntomas), días de hospitalización y exámenes de laboratorio (PCR, LDH, dímero D: DD y recuento absoluto de linfocitos). En todos ellos se registró el peor valor de las primeras $72 \mathrm{~h}$ desde el ingreso. Se registró además las terapias recibidas, incluyendo oxigenoterapia, corticoide sistémico, Tocilizumab y plasma hiperinmune. En aquellos pacientes que recibieron corticoide sistémico se registró el tipo de droga, días de administración, dosis acumulada y presencia de reacciones adversas.

Como indicador de gravedad se tomó en cuenta el tipo de soporte respiratorio requerido por cada paciente, clasificando a los pacientes en 1 de 5 grupos: grupo 1 ( $\sin$ requerimiento de oxigenoterapia), grupo 2 (oxigenoterapia por naricera), grupo 3 (oxigenoterapia por mascarilla de tipo Venturi), grupo 4 (soporte con cánula nasal de alto flujo (CNAF) y/o ventilación mecánica no invasiva (VMNI)) y grupo 5 (soporte con ventilación mecánica invasiva (VMI)). Se considera que los pacientes del grupo 3,4 y 5 son de mayor gravedad y tienen peor pronóstico.

Las tomografías computadas de tórax (TC tó- 
rax) fueron obtenidas con equipos Siemens Somaton Sensation y Siemens Flash, con los pacientes en decúbito supino, en inspiración profunda. Las imágenes fueron reconstruidas con $1 \mathrm{~mm}$ de espesor para la ventana de pulmón y $3 \mathrm{~mm}$ de espesor para la ventana mediastínica, y de $1 \mathrm{~mm}$ en casos de sospecha de tromboembolismo pulmonar. El análisis del patrón tomográfico fue realizado por un único radiólogo especialista.

Los hallazgos imagenológicos fueron clasificados según el patrón predominante (de un total de 7 patrones posibles): 1) Vidrio Esmerilado (VE); 2) VE con patrón en empedrado ("crazy paving”); 3) mixto (VE y condensación); 4) condensación; 5) condensación con pérdida de volumen; 6) patrón de bandas subpleurales, atelectasias y dilatación bronquial y 7) patrón típico de distrés respiratorio agudo (SDRA). Adicionalmente se indicaban los casos que presentaban hallazgos típicos de Neumonía en Organización (OP).

Para evaluar la extensión de las alteraciones en la TC, se utilizó una clasificación de 0-4 puntos según el porcentaje de pulmón comprometido medido en tres niveles: sobre la carina, entre carina y límite superior del ligamento pulmonar inferior, y bajo el ligamento pulmonar inferior. Las categorías posibles fueron: 0 (normal), 1 (extensión menor a 25\%), 2 (extensión entre 25$50 \%$ ), 3 (extensión entre 50-75\%), 4 (extensión mayor a $75 \%$ ). Considerando que cada pulmón fue divido en tres niveles, y con un puntaje máximo de 4 para cada nivel, el puntaje osciló entre 0 y 24 puntos $^{8}$.

\section{Análisis estadístico}

El tamaño muestral fue igual al número de pacientes hospitalizados registrados y analizados. Las variables continuas se presentan como mediana y rango, mientras que las variables categóricas son expresadas como número de pacientes $\mathrm{y}$ porcentaje, con un $95 \%$ de intervalo de confianza.

En el análisis estadístico para identificar factores de riesgo de mayor gravedad, se utilizó test $\chi^{2}$ de Pearson o test de Fisher para realizar comparación de variables categóricas y test de Mann-Whitney para realizar comparación de variables continuas.

\section{Resultados}

Un total de 164 pacientes fueron incluidos en el estudio, con una mediana de edad de 57 años (rango: 21 a 89) y $68 \%$ son de género masculino (Tabla 1). Setenta pacientes (43\%) tienen 60 años o más. Hubo una mediana de 7 días de síntomas previo al ingreso (rango: 1 a 23) y los días de hospitalización tuvieron una mediana de 9 días (rango: 2 a 69). Del total de pacientes, 121 de 164 (74\%) tuvieron al menos una comorbilidad y de estas las más comunes fueron Obesidad (41\%), HTA (34\%), Diabetes (26\%) y enfermedad pulmonar crónica (14\%). El porcentaje de obesos fue significativamente mayor en pacientes menores de 60 años $(\mathrm{p}=0,026)$.

Con respecto a los exámenes de laboratorio, el recuento absoluto de linfocitos tuvo una mediana de 750/ $\mu \mathrm{L}$ (rango: 70 a 2.980), los niveles plasmáticos de LDH una de $336 \mathrm{U} / \mathrm{L}$ (rango: 156 a 1.079), la PCR alcanzó una de $89 \mathrm{mg} / \mathrm{L}$ (rango: 1 a 422), los niveles de DD una media de $0,4 \mu \mathrm{g} /$ $\mathrm{ml}$ (rango: 0,1 a 7,9) y los niveles de ferritina tuvieron una de $1.147 \mathrm{ng} / \mathrm{ml}$ (rango: 47 a 7.199). Los valores normales en nuestro laboratorio son: recuento absoluto de linfocitos (1.000-4.000/ $\mu \mathrm{L})$; LDH plasmática (135-225 U/L); PCR (0-5 $\mathrm{mg} / \mathrm{L})$; niveles de $\mathrm{DD}(0-0,5 \mu \mathrm{g} / \mathrm{ml})$ y ferritina plasmática $(30-400 \mathrm{ng} / \mathrm{mL})$.

En cuanto a los requerimientos de oxígeno sólo 7 pacientes $(4 \%)$ se mantuvieron sin oxigenoterapia durante su hospitalización. $82(50 \%)$ requirieron oxigenoterapia por naricera, $5(3 \%)$ lo recibieron por mascarilla Venturi, 51 (31\%) requieren soporte ventilatorio con $\mathrm{CNAF} y / 0$

Tabla 1. Características basales de los 164 pacientes hospitalizados por COVID-19

\begin{tabular}{|lc|}
\hline Demográficas & n (\%) \\
\hline Edad mediana (rango) & $57(21-89)$ \\
$\geq 60$ años & $70(43)$ \\
$<60$ años & $94(57)$ \\
Género & \\
Masculino & $111(68)$ \\
Femenino & $53(32)$ \\
Comorbilidades & \\
Hipertensión arterial & $56(34)$ \\
Obesidad (IMC $>30)$ & $68(41)$ \\
$\geq 60$ años & $22(31)$ \\
$<60$ años & $46(49)$ \\
Diabetes Mellitus & $43(26)$ \\
Cardiopatía & $14(9)$ \\
Enfermedad pulmonar crónica & $23(14)$ \\
Asma bronquial & $12(7)$ \\
EPOC & $10(6)$ \\
Enfisema/fibrosis & $1(1)$ \\
Tabaquismo & $9(5)$ \\
Cáncer activo $(<1$ año) & $5(3)$ \\
\hline
\end{tabular}




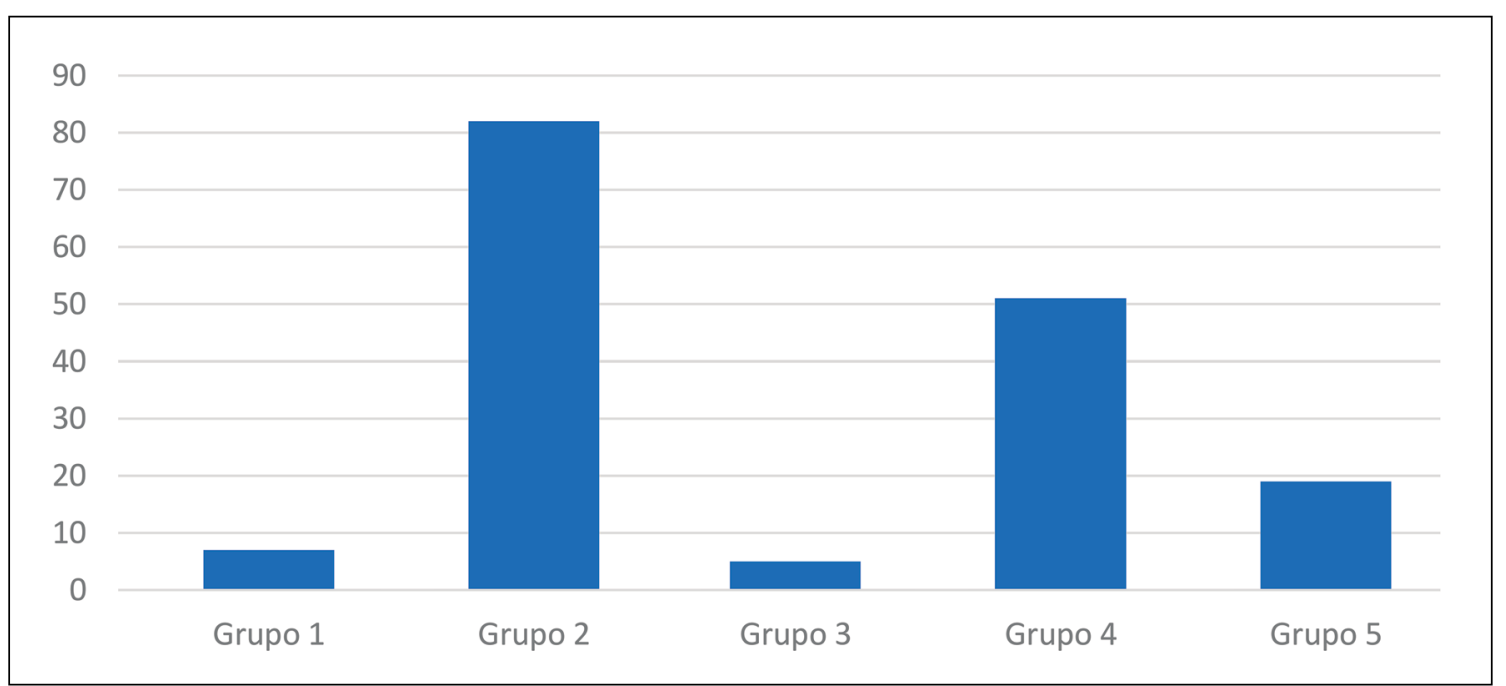

Figura 1. Distribución de los pacientes COVID-19 según tipo de soporte ventilatorio requerido durante su hospitalización. Las cifras de la abscisa representan el número de pacientes. Grupo 1: sin requerimiento de oxigenoterapia; Grupo 2: oxigenoterapia por naricera; Grupo 3: oxigenoterapia por mascarilla de tipo Venturi; Grupo 4: soporte con CNAF (cánula nasal de alto flujo) y/o VMNI (ventilación mecánica no invasiva); Grupo 5: soporte con VMI (ventilación mecánica invasiva).

VMNI y 19 (12\%) requirieren conexión a ventilación mecánica invasiva (Figura 1). Tomados en conjunto los pacientes de los grupos 2, 3, 4 y 5; tienen una mediana de días de hospitalización de 17 (rango: 6 a 69 días).

Del total de pacientes hospitalizados, 12 de $164(7 \%)$ tuvieron tromboembolismo pulmonar confirmado con angioTAC de tórax. Ocho de estos 12 pacientes $(67 \%)$ corresponden a los grupos 3,4 y 5 de tipo de soporte respiratorio y eran por lo tanto pacientes más graves.

Con respecto a las terapias recibidas durante la hospitalización, del total de pacientes, 139 (85\%) recibieron corticoides sistémicos, 22 (13\%) fue- ron tratados con plasma hiperinmune, 7 (4\%) recibieron Tocilizumab y otros 7 (4\%) fueron tratados con plasma hiperinmune y Tocilizumab. La Tabla 2 resume el tipo de corticoide utilizado, la dosis acumulada y los días de tratamiento. Al momento del alta 103 pacientes $(63 \%)$ recibieron la prescripción de continuar con corticoide oral.

En cuanto a efectos adversos secundarios al uso de corticoides, en 82 de los 139 (59\%) no hubo ninguno reportado, mientras que 46 (33\%) tuvieron hiperglicemia que requirió manejo con insulinoterapia, 4 (3\%) se complicaron con sobreinfección bacteriana y 7 (5\%) tuvieron ambas complicaciones.

Tabla 2. Descripción de la terapia con corticoides sistémicos recibidos por 139 pacientes durante la hospitalización por COVID-19

\begin{tabular}{|c|c|c|c|}
\hline \multirow[t]{2}{*}{ Variable } & \multicolumn{3}{|c|}{ Tipo de corticoide recibido } \\
\hline & Metilprednisolona & Dexametasona & Prednisona \\
\hline $\mathrm{n}(\%)$ & $106(76)$ & $31(22)$ & $2(1)$ \\
\hline \multicolumn{4}{|c|}{ Días de terapia: n (\%) } \\
\hline 1-5 días & $58(55)$ & $18(58)$ & $1(50)$ \\
\hline 6-10 días & $48(45)$ & $13(42)$ & $1(50)$ \\
\hline \multicolumn{4}{|c|}{ Dosis acumulada (mg) } \\
\hline 1-5 días & $400(\mathrm{r} 200-1.250)$ & $100(\mathrm{r} 20-155)$ & 180 \\
\hline 6-10 días & 800 (r 200-2.200) & $160($ ( $80-680)$ & 360 \\
\hline \multicolumn{4}{|c|}{ Más de 1 pulso } \\
\hline No & $83(78)$ & $26(84)$ & $2(100)$ \\
\hline Sí & $23(22)$ & $5(16)$ & $0(0)$ \\
\hline
\end{tabular}


Se analizaron posibles variables predictoras de gravedad (definida esta por necesidad de un mayor soporte respiratorio), de las cuales fueron estadísticamente significativas el antecedente de HTA $(p=0,001)$, Diabetes mellitus $(p=0,001)$ y Obesidad. $(p=0,002)$. No hubo diferencias al analizar el grupo de pacientes de 60 años o más (Tabla 3).

Del punto de vista radiológico, del total de pacientes hospitalizados, 150 (91\%) tienen al menos una TC de tórax durante su estadía. La Figura 2 muestra imágenes de los patrones considerados

Tabla 3. Análisis de variables predictoras de gravedad de 164 pacientes hospitalizados por COVID-19

\begin{tabular}{|lccc|}
\hline Variable & Grupos 1-2 & $\begin{array}{c}\text { Tipo de soporte respiratorio } \\
\text { Grupos 3-4-5 }\end{array}$ & Valor de p \\
\hline Edad $\geq 60$ años & $34(49 \%)$ & $36(51 \%)$ & 0,21 \\
Hipertensión arterial & $20(36 \%)$ & $36(64 \%)$ & 0,001 \\
Obesidad & $27(40 \%)$ & $41(60 \%)$ & 0,002 \\
Diabetes mellitus & $12(28 \%)$ & $31(72 \%)$ & 0,001 \\
\hline
\end{tabular}

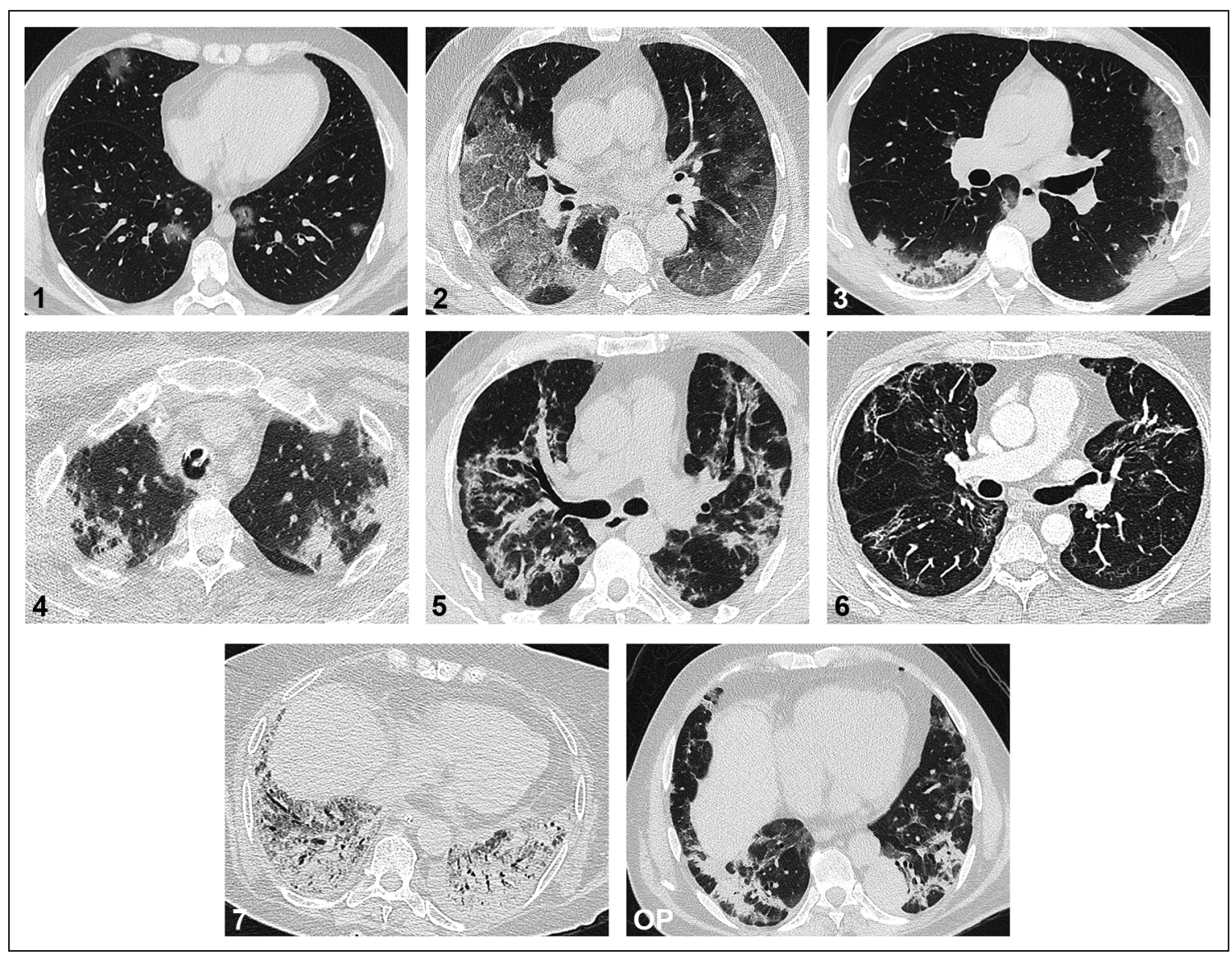

Figura 2. Imágenes de TC de los 7 patrones considerados para definir compromiso radiológico por COVID-19 y TC con hallazgos típicos de OP. 1) VE puro: áreas en VE de configuración nodular, bilaterales. 2) VE y empedrado ("Crazy Paving"): áreas en VE con engrosamiento septal sobrepuesto, que determina patrón en empedrado, bilateral. 3) Mixto : áreas en VE y otras de condensación bilaterales. 4) Condensación: áreas fudamentalmente de condensación pulmonares bilaterales. 5) Condensación con pérdida de volumen: áreas de condensación asociadas a pérdida de volumen, en algunas zonas con aspecto de neumonía en organización. 6) Bandas subpleurales, atelectasias y dilatación bronquial: bandas densas y de atelectasia bilaterales, peribronquiales y periféricas. 7) Distrés respiratorio: extensas áreas de condensación, con broncograma aéreo, simétricas y más densas en zonas dependientes. OP) Neumonía en organización: áreas de condensación asociadas a pérdida de volumen peribronquiales bilaterales. 
en este trabajo y las Figuras 3, 4 y 5 resumen los patrones tomográficos encontrados. La primera TC fue realizada con una mediana de 10 días desde el inicio de los síntomas (rango 0 a 35) y los patrones predominantes fueron el mixto $(35 \%)$, luego el de VE puro (28\%) y VE con "crazy paving" (18\%). 57 pacientes (35\%) tienen más de una TC durante su hospitalización. En la segunda TC (con una mediana de 18 días desde el inicio de los síntomas) los patrones predominantes son: condensación con pérdida de volumen (44\%), VE puro (24\%) y VE con "crazy paving" $(20 \%)$. La prevalencia de OP fue aumentando desde el primer TC $(9 \%)$ hasta el segundo y tercer estudio ( $33 \%$ en cada uno). La extensión del compromiso tuvo también un aumento desde el primer TC (12 puntos) hasta 14 y 15 puntos en la $2^{\mathrm{a}}$ y $3^{\mathrm{a}} \mathrm{TC}$, respectivamente.
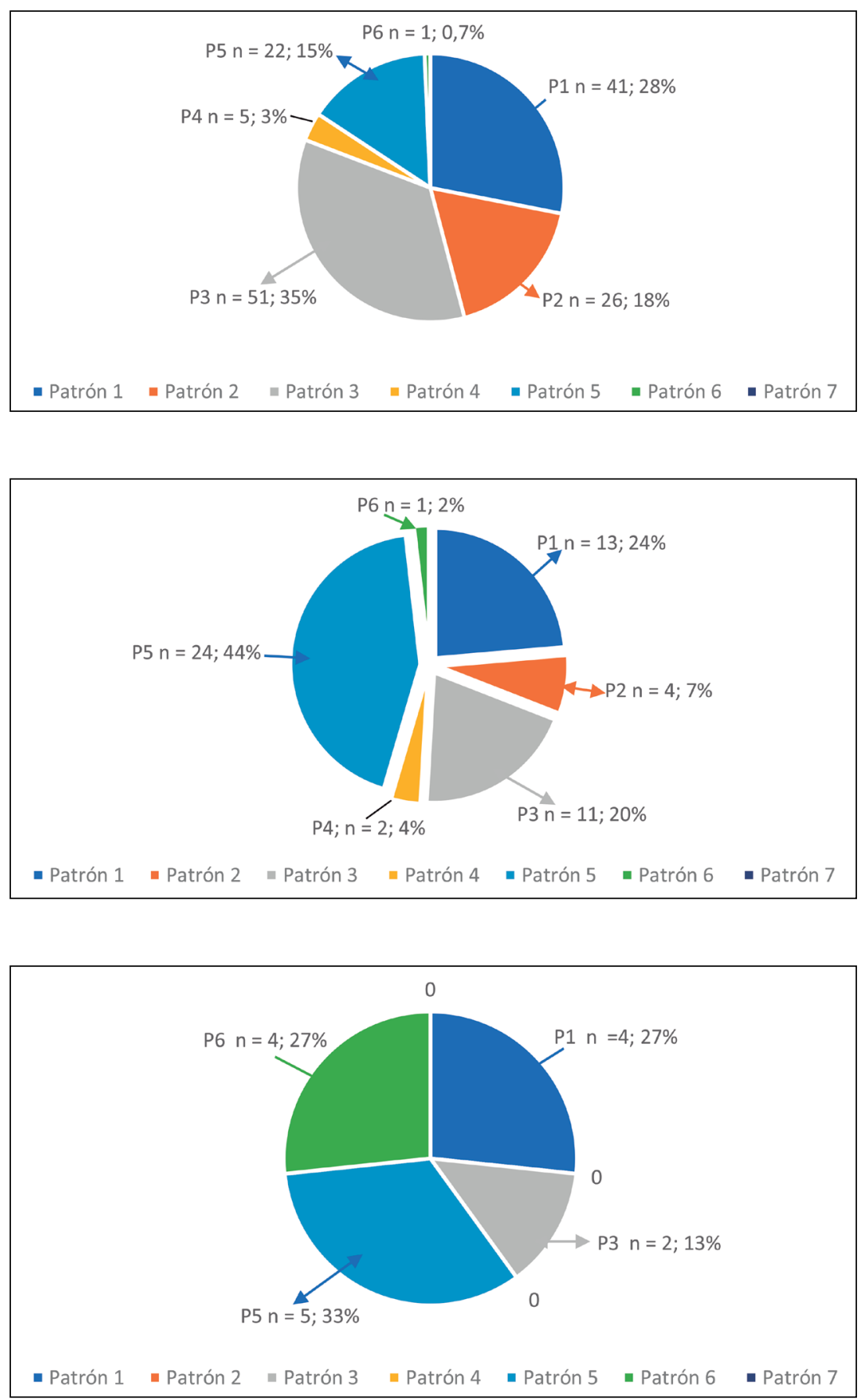

Figura 3. Patrones tomográficos en TC de tórax de ingreso $(n=146)$. Las cifras corresponden al número de pacientes y $\%$ que presentan cada patrón $(\mathrm{P})$ predominante (ver imágenes de Figura 2).

Figura 4. Patrones tomográficos en segunda TC de tórax $(\mathrm{n}=55)$ desde el ingreso. Las cifras corresponden al numero de pacientes y $\%$ que presentan cada patrón $(\mathrm{P})$ predominante (ver imágenes de Figura 2).

Figura 5. Patrones tomográficos en tercera TC de tórax $(\mathrm{n}=15)$ desde el ingreso. Las cifras corresponden al número y $\%$ de pacientes que presentan cada patrón $(\mathrm{P})$ predominante (ver imágenes de Figura 2). 


\section{Discusión}

Distintas publicaciones de series de casos de pacientes hospitalizados por COVID-19 concluyen un predominio de pacientes de género masculino. En una cohorte de más de 1.000 pacientes hospitalizados, publicada por Guan ${ }^{8}, 58 \%$ eran de género masculino con una mediana de edad de 47 años (rango 35 a 58). En otra serie publicada por Giacomelli ${ }^{9}, 69 \%$ eran de género masculino y la mediana de edad era de 61 años (rango 50 a 72). Asimismo, se describen como comorbilidades más comunes la hipertensión arterial (HTA), diabetes mellitus y obesidad ${ }^{10}$.

En el presente trabajo se realizó una caracterización clínica y tomográfica de una población de pacientes que requirieron hospitalización por COVID-19. Los hallazgos del estudio muestran características similares de nuestra población a lo descrito en varias series de casos extranjeras. Se confirma que existe un predominio de pacientes de género masculino y la mayoría de ellos presentan al menos una comorbilidad. Varias comorbilidades muestran asociación con mayor gravedad del cuadro, destacando la presencia de HTA, diabetes y obesidad. Esta última además presente en una cantidad significativa de pacientes $<60$ años. No se logró demostrar en esta serie una asociación entre gravedad y edad $\geq 60$ años.

Con respecto a los hallazgos de laboratorio, una revisión sistemática de 19 estudios, en pacientes con COVID-19, 88\% de ellos hospitalizados $^{11}$, reporta que los hallazgos más frecuentes de laboratorio son: elevación de la proteína $\mathrm{C}$ reactiva $(\mathrm{PCR})$ en más del $60 \%$ de los pacientes $\mathrm{y}$ de lactato deshidrogenasa (LDH) en el 50 a $60 \%$ de los pacientes. En exámenes hematológicos destaca la linfopenia, la cual se presenta en el 63 a $83 \%$ de los hospitalizados ${ }^{12}$. Estos hallazgos son inespecíficos y se observan en neumonías de otras etiologías. Este mismo grupo de pacientes frecuentemente muestra un perfil de laboratorio compatible con hipercoagulabilidad ${ }^{13,14}$ y una alta prevalencia de eventos trombóticos, tanto arteriales como venosos. Una serie de pacientes hospitalizados en $\mathrm{China}^{15}$ muestra que el $43 \%$ de los pacientes no graves y un $60 \%$ de los pacientes graves en intensivo tienen niveles elevados de dímero D (DD). En otra serie de $\operatorname{casos}^{16}$ los niveles elevados de dímero D (DD) $(>1 \mu \mathrm{g} / \mathrm{ml})$ al ingreso, se asocian a aumento de la mortalidad intrahospitalaria. En cuanto a exámenes de laboratorio nuestros datos también concuerdan con lo descrito en las series internacionales publicadas.
La prevalencia de tromboembolismo pulmonar agudo en nuestra serie fue del $7 \%$, sin embargo, esta aumenta a $21 \%$ al analizar el subgrupo de pacientes que requirieron soporte ventilatorio invasivo.

Según el consenso de la Sociedad Radiológica de Norteamérica (RSNA) publicado en marzo del año $2020^{17}$, los hallazgos típicos en COVID-19 son áreas en vidrio esmerilado (VE) con o sin consolidación o patrón en empedrado ("crazy paving"), bilaterales periféricas, o multifocales redondeadas, así como signos sugerentes de neumonia en organización (OP). Asimismo, se ha descrito la evolución de los hallazgos imagenológicos en el curso de la enfermedad $^{18}$. El patrón inicial del compromiso pulmonar suele ser VE, generalmente periférico, basal, y en algunas ocasiones nodular. Progresa a consolidación, siendo el patrón mixto de VE y consolidación lo más frecuente en la fase temprana de la enfermedad. El peak o cúspide de las alteraciones suele verse entre los 6 y 11 días de iniciados los síntomas. Posteriormente aparece distorsión del parénquima y alteraciones que pueden sugerir OP, entre los días 12 y 17 . En las fases tardías vuelve a aparecer VE, se cree como absorción o recuperación de la enfermedad. En nuestra revisión retrospectiva los patrones descritos en las TC de tórax, revisadas por un único radiólogo especialista, también son consistentes a lo descrito en la literatura, siendo las opacidades de VE (puro o con empedrado) el hallazgo más común durante los primeros 10 días de enfermedad. Luego de la segunda semana predominan aquellos patrones que presentan condensación, con un $1 / 3$ de los pacientes con signos típicos de neumonía en organización (OP).

Con respecto a las terapias recibidas destaca el alto porcentaje de pacientes que fueron tratados con corticoides sistémicos (en su gran mayoría por vía endovenosa) lo cual se apoya en el resultado del estudio Recovery ${ }^{19}$ en el cual la utilización de Dexametasona disminuyó la mortalidad a 28 días de 24,6 a 21,6\%, con un mayor beneficio en pacientes con síntomas durante más de 7 días y en aquellos que requirieron ventilación mecánica. En nuestro trabajo se muestra que el uso de corticoides sistémicos fue en general seguro, con casi el $60 \%$ de los pacientes sin un efecto adverso demostrado. No obstante, destaca también el alto porcentaje de pacientes $(63 \%)$ en los cuales se prescribe corticoides al momento del alta. Considerando que solo el $33 \%$ de los pacientes presentan un patrón tomográfico típico de OP, se 
podría considerar que existe una sobreutilización de la terapia corticoidal por tiempo prolongado.

Nuestro trabajo tiene varias limitaciones: en primer lugar su diseño observacional y retrospectivo, que conlleva algunas dificultades en la recolección de los datos a registrar y en segundo lugar el tamaño muestral el cual pudo ser mayor y se vio afectado por la gran carga asistencial a la cual estuvo sometido el personal médico de especialidad que participó en esta revisión.

Como fortalezas destaca la utilización en nuestra institución de un algoritmo de manejo para los pacientes hospitalizados con COVID-19, el cual fue discutido y diseñado de manera multidisciplinaria por un comité de expertos en el tema. Otra fortaleza radica en que la revisión de las imágenes de $\mathrm{TC}$ de tórax fue realizada por un único radiólogo experto (P. Bitar).

\section{Bibliografía}

1.- JOHN HOPKINS, UNIVERSITY \& MEDICINE, Coronavirus Resource Center. Disponible en: https:// coronavirus.jhu.edu/map.html.

2.- MYERS L, PARODI S, ESCOBAR G, LIU V. Characteristics of hospitalized adults with COVID-19 in an integrated health care system in California. JAMA 2020; 323 (21): 2195-8.

3.- YANG X, YU Y, XU J, SHU H, XIA J, LIU H, et al. Clinical course and outcomes of critically ill patients with SARS-CoV-2 pneumonia in Wuhan, China: a single-centered, retrospective, observational study. Lancet Respir Med 2020; 2213-2600(20)30079-5.

4.- GRASSELLI G, ZANGRILLO A, ZANELLA A, ANTONELLI M, CABRINI L, CASTELLI A, et al. Baseline Characteristics and Outcomes of 1591 Patients Infected With SARS-CoV-2 Admitted to ICUs of the Lombardy Region, Italy. JAMA 2020; 323 (16): 1574 81. doi: 10.1001/jama.2020.5394.

5.- GOYAL PJ, CHOI J, PINHEIRO L, SCHENCK E, CHEN R, JABRI A, et al. Clinical Characteristics of Covid-19 in New York City. Correspondence, N Engl J Med 2020; 382 (24): 2372-4. doi: 10.1056/NEJMc2010419. Epub 2020 Apr 17.

6.- WANG D, HU B, HU C, ZHU F, LIU X, ZHANG J, et al. Clinical Characteristics of 138 Hospitalized Patients With 2019 Novel Coronavirus-Infected Pneumonia in Wuhan, China. JAMA 2020; 323 (11): 1061-9. doi: 10.1001/jama.2020.1585.

7.- WHO Clinical management of severe acute respiratory infection when novel coronavirus $(\mathrm{nCoV})$ infection is suspected: interim guidance, 25 January 2020. Published January 25, 2020. Accessed March 30, 2020. Disponible en: https://apps.who.int/ iris/handle/10665/330854
8.- GUAN W, NI Z, HU Y, LIANG W, OU C, HE J, et al. Clinical Characteristics of Coronavirus Disease 2019 in China. N Engl J Med 2020; 382 (18): 1708-20. doi: 10.1056/NEJMoa2002032.

9.- GiACOMELli A, Ridolfo A, MilazZO L, ORENI L, BERNACCHIA D, SIANO M, et al. 30day mortality in patients hospitalized with COVID-19 during the first wave of the Italian epidemic: a prospective cohort study. Pharmacological Research 2020; 1043 6618(20)31239-1.

10.- RICHARDSON S, HIRSCH J, NARASIMHAN M, CRAWFORD J, MCGINN T, DAVIDSON K, et al. Presenting Characteristics, Comorbidities, and Outcomes Among 5700 Patients Hospitalized With COVID-19 in the New York City Area. JAMA 2020; 323 (20): 2052-9. doi: 10.1001/jama.2020.6775.

11.- RODRÍGUEZ-MORALES AJ, CARDONA-OSPINA JA, GUTIÉRREZ-OCAMPO E, VILLAMIZARPEÑA R, HOLGUIN-RIVERA Y, ESCALERAANTEZANA JP, et al. Latin American Network of Coronavirus Disease 2019-COVID-19 Research (LANCOVID-19). Electronic address: https://www. lancovid.org. Clinical, laboratory and imaging features of COVID-19: A systematic review and meta-analysis. Travel Med Infect Dis 2020; 34: 101623. doi: 10.1016/j. tmaid.2020.101623.

12.- HUANG CH, WANG Y, LI X, REN L, ZHAO J, HU $\mathrm{Y}$, et al. Clinical features of patients infected with 2019 novel coronavirus in Wuhan, China. Lancet 2020; 395 (10223): 497-506. doi: 10.1016/S0140-6736(20)301835 .

13.- RANUCCI M, BALLOTTA A, DI DEDDA U, BAYSHNIKOVA E, DEI POLI M, RESTA M, et al. The procoagulant pattern of patients with Covid-19 acute respiratory distress syndrome. J Thromb Haemost 2020; 18: 1747-51.

14.- PANIGADA $\mathrm{M}$, BOTTINO N, TAGLIABUE P, GRASSELLI G, NOVEMBRINO C, CHANTARANGKUL V, et al. Hypercoagulability of COVID-19 patients in intensive care unit. A report of thromboelastography findings and other parameters of hemostasis. J Thromb Haemost 2020; 18 (7): 1738-42.

15.- ZHOU F, YU T, DU R, FAN G, LIU Y, LIU Z, et al. Clinical course and risk factors for mortality of adult inpatients with COVID-19 in Wuhan, China: A retrospective cohort study. Lancet 2020; 395: 105462.

16.- TANG N, LI D, WANG X, SUN Z. Abnormal coagulation parameters are associated with poor prognosis in patients with novel coronavirus pneumonia. J Thromb Haemost 2020; 18 (4): 844-9.

17.- SIMPSON S, KAY FU, ABBARA S, BHALLA S, CHUNG JH, CHUNG M, et al. Radiological Society of North America Expert Consensus Statement on Reporting Chest CT Findings Related to COVID-19. 
Endorsed by the Society of Thoracic Radiology, the American College of Radiology, and RSNA. Disponible en: https://doi.org/10.1148/ryct.2020200152.

18.- WANG Y, DONG C, HU Y, LI C, REN Q, ZHANG $\mathrm{X}$, et al. Temporal Changes of CT Findings in 90 Patients with COVID-19 Pneumonia: A Longitudinal
Study, Radiology 2020; 296: E55-E64.

19.- HORBY P, LIM W, EMBERSON J, MAFHAM J, BELL J, LINSELL L, et al. Effect of dexamethasone in hospitalized patients with COVID-19: preliminary report. N Engl J Med 2020; 383 (21): 2030-40. doi: 10.1056/NEJMoa2022926.

Correspondencia a:

Dr. Cristian Deza Espinosa

Clínica Las Condes

Estoril 450 Comuna Las Condes

Santiago, Chile.

Email: cristiandezaesp@gmail.com 